\title{
Identificación de un generador de inducción doblemente alimentado basado en el filtro de Kalman en presencia de datos espurios
}

\section{Doubly fed induction generator identification based on Kalman filtering in the presence of spurious data}

\author{
Carlos D. Zuluaga*, Eduardo Giraldo*§ \\ * Programa de Ingeniería Eléctrica, Universidad Tecnológica de Pereira. Pereira, Colombia \\ §cardazu@utp.edu.co,egiraldos@utp.edu.co
}

(Recibido: Octubre 31 de 2011 - Aceptado: Mayo 02 de 2013)

\begin{abstract}
Resumen:
Este documento presenta una metodología de identificación de un generador de inducción doblemente alimentado (DFIG) en presencia de datos espurios. El DFIG es ampliamente usado en la producción de energía eléctrica a partir del viento; un problema en el control de estas máquinas, es el cambio en los parámetros del sistema, haciendo que el esquema de control no tenga un óptimo desempeño. Además, si el sistema sensorial no es confiable, se puede incurrir en que las mediciones contengan datos espurios. Para llevar a cabo la identificación se emplea tres filtros de Kalman secuenciales, dos de ellos corresponden al filtro de Kalman dual, el otro corresponde al filtro de Kalman de estadística robusta. La metodología se implementó en Matlab, mostrando que la técnica no se ve afectada por datos espurios, obteniendo errores residuales menores al 1.2\% para la identificación del DFIG en presencia de estos datos.
\end{abstract}

Palabras claves: Datos espurios, DFIG, filtro de Kalman, identificación de sistemas.

\begin{abstract}
This paper presents a methodology for parameter identification of a doubly fed induction generator (DFIG) in the presence of spurious data. DFIG is widely used in the electrical energy production using wind; one problem that the control system for these machines faces is the variability in the parameter values, and optimal performance for this control system is then hard to achieve. Besides, if the sensory system is not reliable, incurring in measurements with high uncertainty may be very common. To perform the parameter identification, three sequential Kalman filters are used, two of them are the dual Kalman filter, and another is the robust statistic Kalman filter. The methodology was implemented in Matlab, showing that the method is not affected by these data, obtaining residual errors smaller than $1.2 \%$ for the DFIG identification in the presence of these data.
\end{abstract}

Keywords: DFIG, Kalman filter, spurious data, , systems identification. 


\section{Introducción}

El generador de inducción doblemente alimentado (DFIG por sus siglas en inglés) es generalmente usado en la producción de energía eléctrica, específicamente en la energía proveniente del viento, la cual usa turbinas eólicas para realizar la conversión, un problema en el control de estas máquinas es el cambio en los parámetros del sistema que hacen que el esquema de control no tenga un buen desempeño (Belfedal et al., 2010). Además de esto se debe agregar que en los sistemas de medida para obtener las variables de interés, para realizar la identificación y el control de estas máquinas, se presentan errores en la medición, originando otro problema al realizar la estimación de parámetros, ya que estas lecturas vienen contaminadas por datos espurios o comúnmente llamados outliers (Ting et al., 2007b).

Un dato espurio, es generalmente definido como "observaciones o medidas que se encuentran por fuera de la distribución de los datos" (Moore \& Mccabe, 1989). La detección de datos espurios ha sido extensamente explorada en minería de datos (Han \& Kamber, 2000), en sistemas de aprendizajes autónomos (Ting, 2009), en sistemas de control automático (Ting et al., 2007b), robots asistidos por computador (Ting et al., 2007a); todas estas aplicaciones han tratado el problema de los datos espurios. Estos datos, ocurren frecuentemente en la práctica y pueden ocasionar serios problemas: un ejemplo catastrófico fue el lanzamiento del cohete Titan IV Centaur en abril de 1999, aparentemente causado por un mal diseño de un filtro en el software de dirección (Eleazer, 2011).

Dentro de los trabajos existentes que realizan la identificación de parámetros de máquinas de inducción se pueden clasificar en dos grandes grupos, los cuales son: la sintonización de parámetros fuera de línea (Schierling, 1988) y la sintonización en línea (Finch et al., 1998). Los estudios que realizan la estimación de los parámetros en línea se subdividen en 4 grandes grupos: el primero son las técnicas basadas en el análisis espectral (Matsuo \& Lipo, 1985; Chai \& Acarnley, 1992; Toliyat \& Hosseiny, 1993), el segundo son los enfoques que utilizan observadores (Loron \& Laliberte, 1993; Zai et al., 1992), el tercero son métodos que se fundamentan en sistemas con modelo de referencia adaptativos (Griva et al., 1997; Ohnishi et al., 1986) y finalmente se tiene otros métodos tal como los actuales desarrollos en la inteligencia artificial (redes neuronales) o sistemas difusos (Hofmann \& Liang, 1995; Bim, 2001). Por otro lado, la identificación fuera de línea (Sumner \& Asher, 1993; Ruff et al., 1994; Barrero et al., 1999) se basa en el desarrollo de pruebas para obtener señales correspondientes al comportamiento de la máquina y luego aplicar un algoritmo de estimación como por ejemplo: mínimos cuadrados recursivos o un enfoque de máxima verosimilitud (Ruff \& Grotstollen, 1993; Borgard et al., 1995; Bertoluzzo et al., 1997), sin embargo se debe notar que la precisión de estos algoritmos dependen sustancialmente de la selección del tiempo de muestreo, error de cuantificación, resolución y buen sistema de medida

Este estudio pretende identificar los parámetros de un DFIG y además garantizar que las cantidades estimadas no estén afectadas por los datos espurios presentes en las mediciones, como lo pueden ser los voltajes en eje directo y en cuadratura de la máquina. Para llevar a cabo este trabajo se combinará tres filtros de Kalman secuenciales, dos de ellos pretenden realizar una estimación dual de la máquina; y el último un preprocesamiento para tratar los datos espurios, estas dos tareas aplicadas dentro del mismo enfoque.

\section{Metodología}

Un esquema simplificado del DFIG para la generación de energía eléctrica a base del viento es presentado en la Figura 1, el cual un convertidor es conectado al rotor de la máquina, para proporcionar la frecuencia necesaria en orden a mantener la frecuencia en el estator en un nivel constante. 


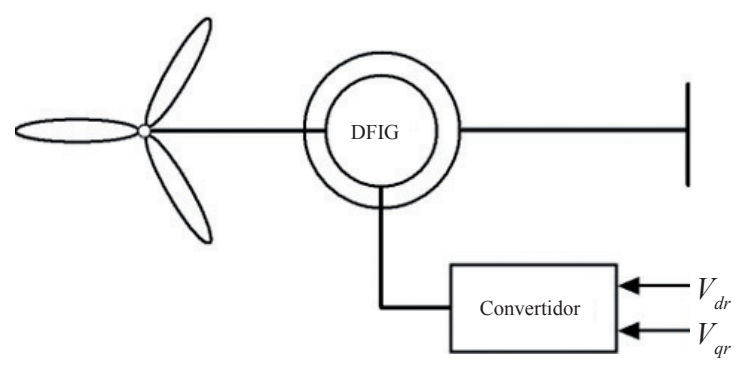

Figura 1. Esquema del sistema experimental.

A continuación se presentará la descripción teórica de los modelos para realizar la identificación de parámetros del DFIG.

\subsection{Modelo del DFIG}

En el contexto de la rotación síncrona, el modelo de un generador de inducción de rotor bobinado está dado por el siguiente conjunto de ecuaciones (Belfedal et al., 2010):

Ecuaciones de las componentes del voltaje en el estator:

$$
\begin{aligned}
& V_{d s}=R_{s} I_{d s}+\frac{d \Phi_{d s}}{d t}-\omega_{s} \Phi_{q s} \\
& V_{q s}=R_{s} I_{q s}+\frac{d \Phi_{q s}}{d t}+\omega_{s} \Phi_{d s}
\end{aligned}
$$

Expresiones para las componentes del voltaje en el rotor

$$
\begin{gathered}
V_{d r}=R_{r} I_{d r}+\frac{d \Phi_{d r}}{d t}-\omega_{r} \Phi_{q r} \\
V_{q r}=R_{r} I_{q r}+\frac{d \Phi_{q r}}{d t}+\omega_{r} \Phi_{d r}
\end{gathered}
$$

Ecuaciones para las componentes del flujo en el estator

$$
\begin{aligned}
& \Phi_{d s}=\mathrm{L}_{s} \mathrm{I}_{d s}+\mathrm{L}_{m} \mathrm{I}_{d r} \\
& \Phi_{q s}=\mathrm{L}_{s} \mathrm{I}_{q s}+\mathrm{L}_{m} \mathrm{I}_{q r}
\end{aligned}
$$

Ecuaciones para las componentes del flujo en el rotor

$$
\begin{aligned}
& \Phi_{d r}=\mathrm{L}_{r} \mathrm{I}_{d r}+\mathrm{L}_{m} \mathrm{I}_{d s} \\
& \Phi_{q r}=\mathrm{L}_{r} \mathrm{I}_{q r}+\mathrm{L}_{m} \mathrm{I}_{q s}
\end{aligned}
$$

Donde $\mathrm{I}_{d s}, \mathrm{I}_{q s}, \mathrm{I}_{d r}, \mathrm{I}_{q r}$, son respectivamente las componentes de la corriente del estator y rotor; $\Phi_{d s}, \Phi_{q s}, \Phi_{d r}, \Phi_{q r}$, son los componentes del flujo del estator y rotor, mientras que $\mathrm{V}_{d s}, \mathrm{~V}_{q s}, \mathrm{~V}_{d r}, \mathrm{~V}_{q r}$, con los voltajes del estator y rotor respectivamente; $\mathrm{R}_{s}, \mathrm{R}_{s}$ son las resistencias del estator y rotos, similarmente $\mathrm{L}_{s}, \mathrm{~L}_{s}$, son las inductancias del estator y rotor; $\mathrm{L}_{m}$ es la inductancia mutua.

El torque electromagnético está dado por la Ec. (9)

$$
\mathrm{T}_{g}=\mathrm{L}_{m}\left(\mathrm{I}_{d r} \mathrm{I}_{q s}-\mathrm{I}_{q r} \mathrm{I}_{d s}\right)
$$

La velocidad mecánica del rotor está representada por la siguiente expresión:

$$
\frac{d \omega_{r}}{d t}=\frac{1}{2 H}\left(\mathrm{~T}_{m}-\mathrm{T}_{g}\right)
$$

donde $\mathrm{T}_{m}$ es el torque de carga que está definido por la turbina conectada al DFIG, $\omega_{r}$ es la velocidad angular del rotor del generador y $H$ es la constante de inercia del generador.

\subsection{Modelo de la turbina}

Teóricamente $\mathrm{T}_{m}$ se puede representar a través de la siguiente ecuación

$$
\mathrm{T}_{m}=\frac{\rho V_{w}^{2} \pi r^{3} C_{T}\left(V_{w}, \omega_{r}\right)}{2}
$$

donde $\rho$ es la densidad del aire, $V_{w}$ es la velocidad del viento, $C_{T}$ es un coeficiente del torque de la turbina, que depende de la relación de $V_{w}, \omega_{\mathrm{r}}$ $\left(\lambda=\omega_{r} r / V_{w}\right)$, para este estudio se modelará $C_{T}$ de la siguiente manera:

$$
C_{T}(\lambda)=c_{1} \lambda^{2}+c_{2} \lambda+c_{3}+\frac{c_{4}}{\lambda}
$$

con coeficientes $\quad c_{1}=0.0013, \quad c_{2}=-0.0439$, $c_{3}=0.4083, c_{4}=-0.6703$ (Endusa et al., 2007).

El modelo del viento ha sido desarrollado por el laboratorio nacional de energía sostenible, RIS $\varnothing$ DTU de la Universidad Técnica de Dinamarca, la velocidad del viento es calculado como un 
promedio de la velocidad del rotor y tiene en cuenta la turbulencia del medio (Sørensen et al., 2002).

\subsection{Síntesis del modelo del DFIG alimentado por turbina eólica.}

El modelo del DFIG presentado en la sección 3.1 puede ser reescrito como sigue (Belfedal et al., 2010)

$$
\begin{gathered}
{\left[\begin{array}{c}
\dot{\Phi}_{d r} \\
\dot{\Phi}_{q r}
\end{array}\right]=\left[\mathbf{A}_{s}\right]\left[\begin{array}{c}
\Phi_{d r} \\
\Phi_{q r}
\end{array}\right]+\left[\mathbf{B}_{s 1}\right]\left[\begin{array}{c}
\mathrm{I}_{d s} \\
\mathrm{I}_{q s}
\end{array}\right]+\left[\mathbf{B}_{s 2}\right]\left[\begin{array}{c}
\mathrm{V}_{d r} \\
\mathrm{~V}_{q r}
\end{array}\right]} \\
{\left[\begin{array}{c}
\mathrm{V}_{d s} \\
\mathrm{~V}_{q s}
\end{array}\right]=\left[\mathbf{C}_{s}\right]\left[\begin{array}{c}
\Phi_{d r} \\
\Phi_{q r}
\end{array}\right]+\left[\mathbf{D}_{s 1}\right]\left[\begin{array}{c}
\mathrm{I}_{d s} \\
\mathrm{I}_{q s}
\end{array}\right]+\left[\mathbf{D}_{s}^{\prime}\right]\left[\begin{array}{c}
\dot{\mathrm{I}}_{d s} \\
\dot{\mathrm{I}}_{q s}
\end{array}\right]+\left[\mathbf{D}_{s 2}\right]\left[\begin{array}{c}
\mathrm{V}_{d r} \\
\mathrm{~V}_{q r}
\end{array}\right]}
\end{gathered}
$$

$\mathrm{I}_{d s} \mathrm{y} \mathrm{I}_{q s}$ son considerados dos variables perturbadas, ya que estas dependen de la carga. Sin embargo, en el contexto de la referencia síncrona, $\mathrm{I}_{d s}$ y $\mathrm{I}_{q s}$ son constantes en estado estable, por lo tanto, sus derivadas se pueden considerar cero, así el modelo del DFIG puede ser estructurado por el siguiente modelo de espacio de estados:

$$
\begin{aligned}
& \mathbf{X}=\left[\mathbf{A}_{s}\right] \mathbf{X}+\left[\mathbf{B}_{s}\right] \mathbf{U} \\
& \mathbf{Y}=\left[\mathbf{C}_{s}\right] \mathbf{X}+\left[\mathbf{D}_{s}\right] \mathbf{U} \\
& \text { donde } \\
& \mathbf{X}=\left[\begin{array}{c}
\Phi_{d r} \\
\Phi_{q r}
\end{array}\right], \mathbf{Y}=\left[\begin{array}{c}
\mathrm{V}_{d s} \\
\mathrm{~V}_{q s}
\end{array}\right], \mathbf{U}=\left[\begin{array}{llll}
\mathrm{I}_{d s} & \mathrm{I}_{q s} & \mathrm{~V}_{d r} & \mathrm{~V}_{q r}
\end{array}\right] \\
& {\left[\mathbf{B}_{s}\right]=\left[\left[\mathbf{B} s_{1}\right]\left[\mathbf{B} s_{2}\right]\right],[\mathbf{D}]=\left[\left[\mathbf{D} s_{1}\right]\left[\mathbf{D} s_{2}\right]\right]}
\end{aligned}
$$

con

$$
\begin{gathered}
{\left[\mathbf{A}_{s}\right]=\left[\begin{array}{rr}
-\frac{R_{r}}{L_{r}} & \omega_{r} \\
-\omega_{r} & -\frac{R_{r}}{L_{r}}
\end{array}\right] \quad\left[\mathbf{B}_{s_{1}}\right]=\frac{R_{r} L_{m}}{L_{r}}\left[\begin{array}{ll}
1 & 0 \\
0 & 1
\end{array}\right] \quad\left[\mathbf{B}_{s_{2}}\right]=\left[\begin{array}{ll}
1 & 0 \\
0 & 1
\end{array}\right]} \\
{\left[\mathbf{C}_{s}\right]=\left[\begin{array}{cc}
\frac{R_{r}}{L_{r}} & \omega \\
\omega & -\frac{R_{r}}{L_{r}}
\end{array}\right] \quad\left[\mathbf{D}_{s_{1}}\right]=\left[\begin{array}{cc}
R_{s}+\frac{L_{m}^{2}}{L_{r}^{2}} R_{r} & -L_{s} \omega_{s} \\
L_{s} \omega_{s} & R_{s}+\frac{L_{m}^{2}}{L_{r}^{2}} R_{r}
\end{array}\right] \quad\left[\mathbf{D}_{s_{2}}\right]=\frac{L_{m}}{L_{r}}\left[\begin{array}{ll}
1 & 0 \\
0 & 1
\end{array}\right]}
\end{gathered}
$$

donde $\mathrm{w}=\mathrm{wr}-\mathrm{ws}$ y ws es la velocidad angular síncrona

\subsection{Filtro de Kalman}

El filtro de Kalman (KF) ofrece una forma de estimar el estado $\mathbf{x}_{k}$ de un modelo en un sistema estocástico lineal de dimensión finita dado por las Ec. (15), (16) y (17), donde $\mathbf{v}_{1}$ y $\mathbf{v}_{2}$ representan entradas de ruido blanco. Si se remueven estas señales de ruido el modelo se reduce a un sistema de espacio de estado determinístico (Goodwin \& Sin, 2009; Ljung, 1999; Sánchez \& Giraldo, 2009).

La estimación del proceso se puede ver como una forma de control por realimentación: el filtro estima el estado en algún tiempo y luego obtiene la realimentación en la forma de las medidas (más ruido). Las ecuaciones para el KF se presentan en dos grupos: actualización de tiempo y actualización de la medida (Anderson \& Moore, 2005).

La señal $\mathbf{v}_{1}$ es el ruido de proceso y $\mathbf{v}_{2}$ es el ruido de la medida u observación, estos son procesos estacionarios de ruido blanco con media cero y no correlacionados.

$$
\begin{array}{r}
\mathbf{x}(k+1)=\mathbf{A x}(\mathrm{k})+\mathbf{B u}(\mathrm{k})+\mathbf{v}_{1}(\mathrm{k}) \\
\mathbf{y}(k)=\mathbf{C x}(k)+\mathbf{D u}(k)+\mathbf{v}_{2}(k)
\end{array}
$$


donde $\mathbf{x}(k)$ son los estados del sistema en un instante $k$, además es una variable aleatoria cuyos valores iniciales para el estado es y covarianza es $\Sigma 0$

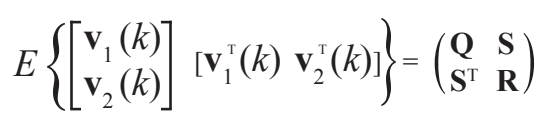

donde $E$ es el valor esperado. Las matrices de covarianza $\mathbf{Q}$ y $\mathbf{R}$ se asumen contantes y la matriz de covarianza $\mathbf{S}$ es cero debido a que no existe correlación entre los ruidos del proceso y medida.

El filtro se basa en las dos interpretaciones siguientes (Sánchez \& Giraldo, 2009):

- Si el ruido es Gaussiano, el filtro presenta la estimación del estado de mínima varianza.

- Si se remueve la hipótesis, el filtro obtiene la estimación del estado de mínima varianza lineal.

El teorema de Kalman se basa en la obtención de una ecuación que estime el estado posterior $\hat{\mathbf{x}}(k)$ como una combinación lineal del estado a priori $\hat{\mathbf{x}}^{-}(k)$, una ponderación de la diferencia entre la medida actual $\mathbf{y}(k)$ y una predicción de la medida $\mathbf{C} \hat{\mathbf{x}}-(k)$, lo anterior se muestra en la Ec. (18)

$$
\hat{\mathbf{x}}(k)=\hat{\mathbf{x}}(k)+\mathbf{K}(k)(\mathbf{y}(k)-\mathbf{C} \hat{\mathbf{x}}(k))
$$

donde $\mathbf{K}(k)$ es la ganancia de Kalman dada por la ecuación Ec. (19)

$$
\mathbf{K}(k)=\boldsymbol{\Sigma}^{-}(k) \mathbf{C}^{\mathrm{T}}\left(\mathbf{C} \boldsymbol{\Sigma}-(k) \mathbf{C}^{T}+\mathbf{R}\right)^{-1}
$$

donde $\Sigma^{-}(k)$ es la matriz de covarianza a priori de la propagación del error de estado.

\subsection{Filtro de Kalman dual}

Suponiendo que se tiene un modelo como el descrito por las Ecs (15) y (16), donde solo se puede medir la señal de entrada $\mathbf{u}(k)$ y la señal de salida $\mathbf{y}(k)$, adicionalmente se tiene información de los estados del sistema, con lo anterior planteado se reescribe el sistema tomando una estructura matricial así:

$$
\begin{gathered}
\mathbf{\Phi}(k)=\left[\begin{array}{l}
\mathbf{x}(k) \\
\mathbf{u}(k)
\end{array}\right], \quad \mathbf{V}(k)=\left[\begin{array}{l}
\mathbf{v}_{1}(k) \\
\mathbf{v}_{2}(k)
\end{array}\right] \\
\mathbf{Y}(k)=\left[\begin{array}{c}
\mathbf{x}(k+1) \\
\mathbf{y}(k)
\end{array}\right], \quad \boldsymbol{\Theta}=\left[\begin{array}{ll}
\mathbf{A} & \mathbf{B} \\
\mathbf{C} & \mathbf{D}
\end{array}\right]
\end{gathered}
$$

Por lo tanto el sistema mostrado en las Ecs (15) y (16) puede ser descrito como

$$
\mathbf{Y}(k)=\boldsymbol{\Theta} \boldsymbol{\Phi}(k)+\mathbf{V}(k)
$$

Para llevar a cabo la estimación de los parámetros $\boldsymbol{\Theta}$ se necesita y se asume tener conocimiento de los estados, para ello se plantea un método de estimación dual, en este procedimiento se han encontrado técnicas que trabajan alternando el uso del modelo para estimar la señal y usar la señal para estimar el modelo. Este proceso puede ser o de forma iterativa o secuencial. El esquema iterativo trabaja repetidamente estimando la señal usando el modelo actual, teniendo todos los datos disponibles, por lo tanto este esquema es usado o es necesariamente restringido a aplicaciones off-line, donde los datos han sido previamente obtenidos para el procesamiento. Por otra parte, el enfoque secuencial usa cada dato individual, tan pronto como esté disponible, así se puede actualizar tanto como la estimación del modelo como la señal que se quiere determinar. Esta característica hace estos algoritmos sean atractivos para aplicaciones on-line o inclusive off-line (Haykin, 2001).

En este estudio se utilizará el enfoque secuencial para realizar la estimación dual, ya que posee una gran ventaja frente al iterativo, además se quiere aprovechar la recursividad del algoritmo del filtro de Kalman.

El filtro de Kalman dual (DKF) se puede resumir como se muestra en el algoritmo 1.

\subsection{Filtro de Kalman de estadística robusta}

Hasta ahora se ha planteado el algoritmo del filtro de Kalman para realizar una estimación de parámetros y estados; partiendo de una 
representación de modelos de espacios de estado se puede proponer una metodología útil y a su vez recursiva para el tratamiento de datos espurios $\mathrm{u}$ outliers en sistemas dinámicos. Varios enfoques han sido utilizados para tratar la sensibilidad de la estimación cuando se presentan estos datos particulares, en sistema dinámicos; algunos de ellos han tratado de realizar un filtro de Kalman que sea robusto ante datos espurios, a través de la sensibilidad del criterio del error cuadrático medio a estos datos (Tukey, 1960; Huber, 1964). Una clase de estudios consideran distribuciones no Gaussianas para las variables aleatorias (Sorenson \& Alspach, 1971; West, 1981; Smith \& West, 1983), ya que las distribuciones Gaussianas multivariadas son susceptibles a datos espurios (Bishop, 2006). Por ejemplo (Meinhold \& Singpurwalla, 1989) usa una distribución t-Student. Sin embargo, la estimación resultante de los parámetros puede ser compleja para sistemas con perturbaciones transitorias. Otras metodologías han intentado modelar el ruido del estado y medida con distribuciones no Gaussianas (Masreliez, 1975; Masreliez \& Martin, 1977; Schick \& Mitter, 1994). Desafortunadamente estos estudios son difíciles de implementar y no proporcionan una buena estimación del vector de estado.

En este estudio se tomara en cuenta la ponderación de la dispersión de los datos de salida del sistema dinámico para detectar $\mathrm{y}$ eliminar los datos espurios presentes en las señales de interés. En orden a tratar estos datos, se define la siguiente hipótesis

$\mathbf{v}_{1} \sim N(0, \mathbf{Q}), \mathbf{v}_{2} \sim N(0, \mathbf{R})$ contaminado $-\varepsilon$

La contaminación $\varepsilon$ en la Ec. (20) indica que la distribución normal, adoptada por el filtro de Kalman estándar, es contaminada por una pequeña fracción $\varepsilon$ (por ejemplo $\varepsilon=0.05$ ) de una distribución simétrica de colas pesadas (o heavy tails), la cual es la fuente de datos espurios.

De lo anterior se hace necesario calcular de nuevo el estado estimado $\hat{\mathbf{x}}(k)$ y puede ser obtenido

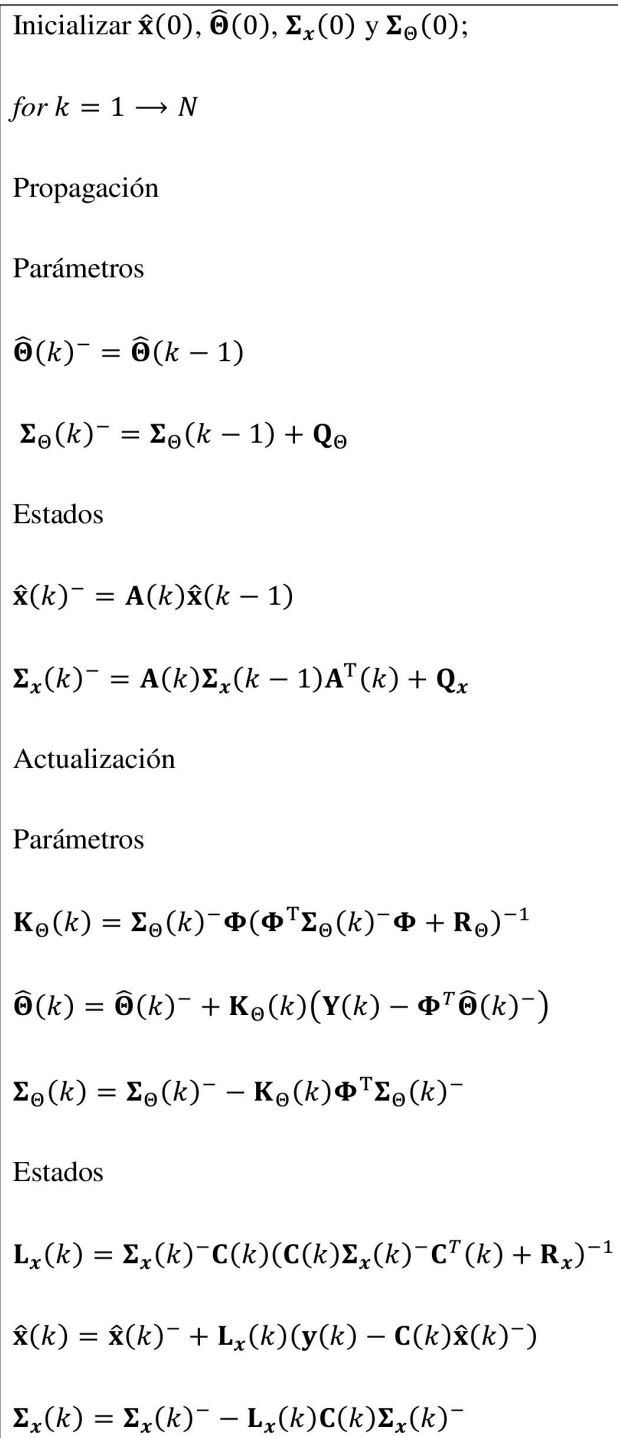

Algoritmo 1: Resumen del filtro de Kalman aplicado a la estimación de estados y parámetros.

minimizando el siguiente funcional:

$$
\begin{array}{r}
\hat{\mathbf{x}}(k)=\operatorname{argmin}\left\{(\hat{\mathbf{x}}(k-1)-\mathbf{x}(k))^{T}(\mathbf{\Sigma}(k-1))^{-1}(\hat{\mathbf{x}}(k-1)-\mathbf{x}(k))\right. \\
\left.+(\mathbf{y}(k)-\mathbf{C x}(k))^{T}(\mathbf{R}(k-1))^{-1}(\mathbf{y}(k)-\mathbf{C x}(k))\right\}
\end{array}
$$

o de forma similar se puede obtener se puede obtener el estado estimado reescribiendo el anterior funcional donde $n$ es la dimensión de los estados y es la dimensión de la salida del sistema, además 


$$
\hat{\mathbf{x}}(k)=\operatorname{argmin}\left\{\sum_{i=1}^{\mathrm{n}}(\mathbf{p} i(k)-\mathbf{a} i(k) \mathbf{x}(k))^{2}+\sum_{j=1}^{\mathrm{m}} \rho\left(\mathbf{s}_{j}(k)-\mathbf{b}_{j}(k) \mathbf{x}(k)\right)^{2}\right\}
$$

También se define $\rho$ como función de costo la cual tiene derivadas, llamadas función de perdida de Huber, usadas en estadística robusta (Huber, 2009). Por dar un ejemplo de cómo es una función psi se tiene lo siguiente

$$
\begin{gathered}
\mathbf{p}_{i}(k)=(\boldsymbol{\Sigma}(k-1))^{-1 / 2} \hat{\mathbf{x}}(k-1), \quad \mathbf{s}_{j}(k)=(\mathbf{R}(k-1))^{-1 / 2} \mathbf{y}(k) \\
\mathbf{a}(k)=(\boldsymbol{\Sigma}(k-1))^{-1 / 2}, \quad \mathbf{b}(k)=(\mathbf{R}(k-1))^{-1 / 2} \mathbf{C} .
\end{gathered}
$$

donde $\operatorname{sgn}(\cdot)$ es la función signo. La Ec (23) es llamada la ecuación psi de Huber (Huber, 2009), esta expresión es muy utilizada para dar estimados robustos, teniendo mínima varianza sobre la distribución contaminada menos favorable. La selección recomendad de en la Ec. (23) es por ejemplo para una contaminación del de los datos (Cipra \& Romera, 1997).

Derivando la Ec. (22) e igualando a cero se tiene

$$
\psi(\mathrm{z})=\left\{\begin{array}{cc}
\mathrm{z}, & |\mathrm{z}| \leq \mathrm{c} \\
\operatorname{csgn}(z) & |\mathrm{z}|>\mathrm{c}
\end{array}\right.
$$

donde en la Ec. (24) las ponderaciones $\omega_{j}(k)$ están definidas por

$$
\sum_{i=1}^{n} \mathbf{a}_{\mathrm{i}}^{\mathrm{T}}(k)\left(\mathbf{p}_{\mathrm{i}}(k)-\mathbf{a}_{\mathrm{i}}(k) \mathbf{x}(k)\right)+\sum_{j=1}^{m} \omega_{j}(k) \mathbf{b}_{j}(k)\left(\mathbf{s}_{j}(k)-\mathbf{b}_{j}(k) \mathbf{x}(k)\right)=0
$$

Con la ayuda de las Ec. (24), (25) y un poco de algebra se llega a que la ganancia de Kalman dada por la Ec. (17) toma la siguiente forma

$$
\begin{gathered}
\omega_{j}(k)=\frac{\psi\left(\mathbf{s}_{j}(k)-\mathbf{b}_{j}(k) \mathbf{x}(k)\right)}{\mathbf{s}_{j}(k)-\mathbf{b}_{j}(k) \mathbf{x}(k)} \\
\mathbf{K}(k)=\boldsymbol{\Sigma}^{-}(k) \mathbf{C}^{\mathrm{T}}\left(\mathbf{C} \boldsymbol{\Sigma}^{-}(k) \mathbf{C}^{\mathrm{T}}+\mathbf{R}^{1 / 2} \mathbf{W}(k) \mathbf{R}^{1 / 2}\right)^{-1}
\end{gathered}
$$

donde $\mathbf{W}(k)=\operatorname{diag}\left\{\omega_{1}(k), \ldots, \omega_{\mathrm{m}}(k)\right\}$.

El tratamiento de datos espurios basado en el filtro de Kalman de estadística robusta (rsKF) se puede observar en el algoritmo 2.

Inicializar $\widehat{\boldsymbol{x}}(0)$ y $\Sigma_{\boldsymbol{x}}(0)$
Propagación
$\hat{\mathbf{x}}(k)^{-}=\mathbf{A}(k) \hat{\mathbf{x}}(k-1)$
$\mathbf{\Sigma}(k)^{-}=\mathbf{A}(k) \mathbf{\Sigma}(k-1) \mathbf{A}^{T}(k)+\mathbf{Q}$
Datos Complementarios
$\mathbf{s}(k)=(\mathbf{R})^{-1 / 2} \mathbf{y}(k)$
$\mathbf{b}(k)=(\mathbf{R})^{-1 / 2} \mathbf{C}(k)$
$\omega_{j}(k)=\frac{\psi\left(\mathbf{s}(k)-\mathbf{b}(k) \hat{\mathbf{x}}(k)^{-}\right)}{\mathbf{s}(k)-\mathbf{b}(k) \hat{\mathbf{x}}(k)^{-}}$
$\mathbf{W}(k)=\operatorname{diag}\left\{\omega_{1}(k), \ldots, \omega_{m}(k)\right\}$
Actualización
$\mathbf{K}(k)=\boldsymbol{\Sigma}^{-}(k) \mathbf{C}^{\mathrm{T}}\left(\mathbf{C} \boldsymbol{\Sigma}^{-}(k) \mathbf{C}^{\mathrm{T}}+\mathbf{R}^{1 / 2} \mathbf{W}(k) \mathbf{R}^{1 / 2}\right)^{-1}$
$\hat{\mathbf{x}}(k)=\hat{\mathbf{x}}(k)^{-}+\mathbf{K}(k)\left(\mathbf{y}(k)-\mathbf{C}(k) \hat{\mathbf{x}}(k)^{-}\right)$
$\boldsymbol{\Sigma}(k)=\mathbf{\Sigma}(k)^{-}-\mathbf{K}(k) \mathbf{C}(k) \boldsymbol{\Sigma}(k)^{-}$

Algoritmo 2: Resumen del filtro de Kalman para el tratamiento de outliers.

\section{Resultados y discusión}

En este estudio, se evalúa el algoritmo de identificación de un generador de inducción doblemente alimentado, para el caso de estar conectado a una turbina eólica; en donde el modelo del viento ha sido realizado por el Laboratorio Nacional de Energía Sostenible, RISØ DTU de la Universidad Técnica de Dinamarca, el cual se puede obtener la velocidad del rotor de la máquina (ver Figura 2), que es considerada como una variable que cambia con el tiempo dentro del sistema de espacio de estados mostrado en la sección (2.2). Para este estudio se utiliza 
una velocidad del viento promedio de $12 \mathrm{~m} / \mathrm{s}$; diámetro del rotor de $15 \mathrm{~m}$; nivel de turbulencia de $4 \%$ y un tiempo de muestreo de $0.1 \mathrm{~s}$. También se utilizó datos aleatorios como señales de entrada al sistema, un ejemplo de estas señales se tiene el voltaje en el rotor y las corrientes en el estator. Las simulaciones fueron realizadas en Matlab R2009a ${ }^{\circledR}$, obteniendo respuestas para los voltajes del estator en eje directo y en cuadratura del DFIG. En las Figuras 3 y 4, se muestra el comportamiento de los voltajes del estator en eje directo y en cuadratura del DFIG, respectivamente, ante variaciones de las señales de control y cuenta además con la variación aleatoria del viento; se muestra la señal de salida y la señal respectiva estimada aplicando la metodología que se basa en el DKF. Se nota que la correspondencia entre estas dos señales es muy similar, por lo tanto se puede notar que el algoritmo de identificación de sistemas realiza de manera adecuada la tarea de estimación.

También de las Figuras 3 y 4 cabe resaltar que el algoritmo de identificación pese a la aleatoriedad del viento, realiza de manera apropiada la estimación de parámetros de sistemas dinámicos, lo que lleva a decir que el DKF es adecuado para abordar el problema de la identificación recursiva de sistemas multivariables acoplados y sirve

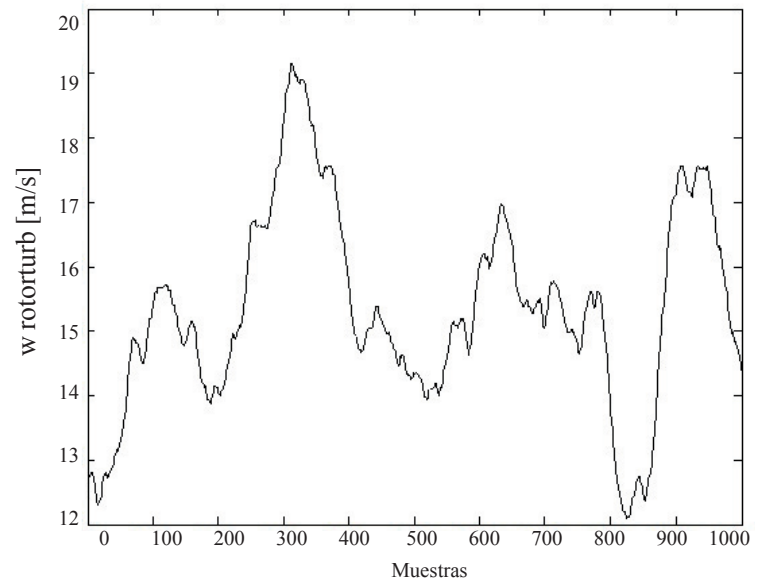

Figura 2. Velocidad de la turbina ante la variación del modelo del viento. Donde $w$ rotorturb es la velocidad de la turbina expresada en $\mathrm{m} / \mathrm{s}$. conjuntamente para aplicaciones de identificación en línea.

Ahora se procede a agregar datos espurios a la señal de salida con el 5\% de probabilidad de todo el conjunto de datos de la señal; esta adición de datos espurios se puede ver como si se tuviera un problema en la medición de estas señales.

En las Figuras 5 y 6 se observa que las señales de salida fueron perturbadas por la presencia de estos datos, también se nota que las señales estimadas han sido afectadas negativamente por estos datos y por lo tanto la identificación fue realizada de manera incorrecta. Ahora bien, se cuenta con el tratamiento de datos espurios.

De las Figuras 7 y 8 se puede resaltar que la señal de salida estimada, por la combinación del filtro de Kalman dual y el filtro de Kalman de estadística robusta (DKF-rsKF), ya no se ve perturbada por los datos espurios presentes en la señal de salida; sin embargo, también se puede notar que la combinación propuesta realiza la identificación no muy rápida en comparación como lo hace el DKF, a pesar de esto se puede concluir que la metodología planteada para la identificación se sistemas considerando datos espurios es adecuada para tratar sistemas dinámicos.

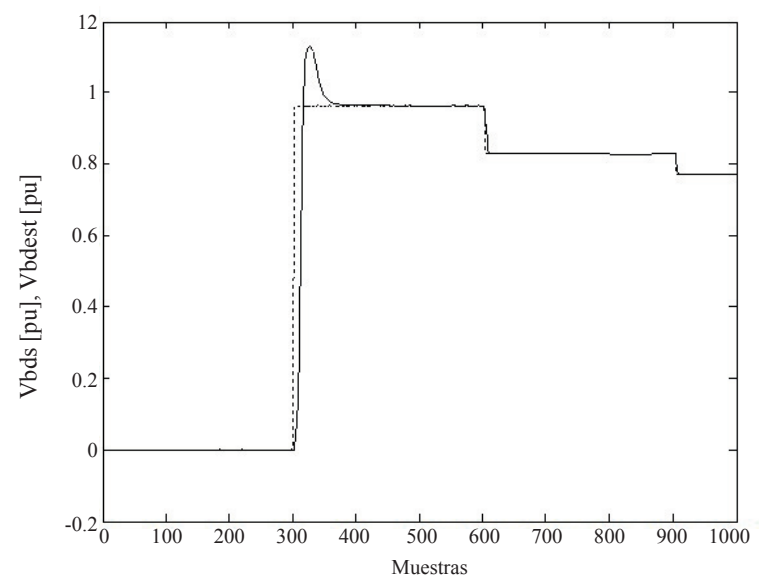

Figura 3. Comportamiento del voltaje en el estator en eje directo sin datos espurios agregados. (----) corresponde al voltaje de la máquina (Vds) y (—) pertenece al voltaje estimada (Vdsest) por el $D K F$. 


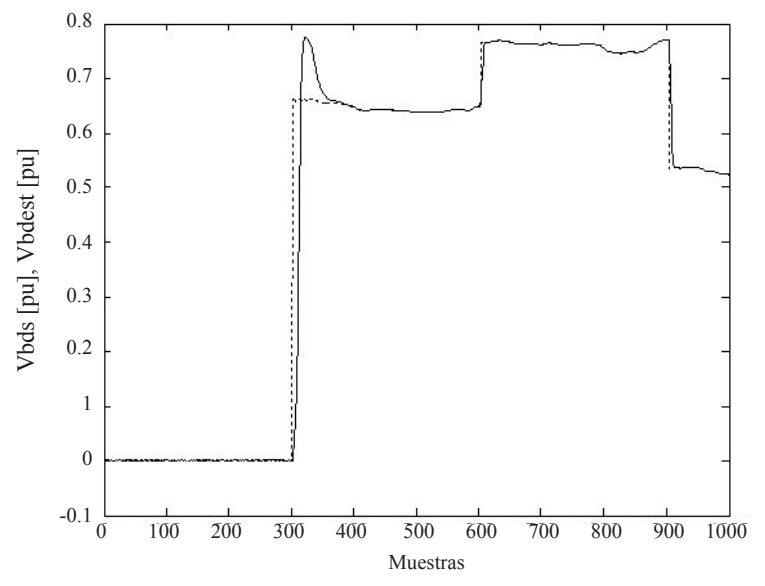

Figura 4. Comportamiento del voltaje en el estator en eje en cuadratura sin datos espurios agregados. (----) corresponde al voltaje de la máquina (Vqs) y (—) pertenece al voltaje estimado (Vdqest) por el DKF.

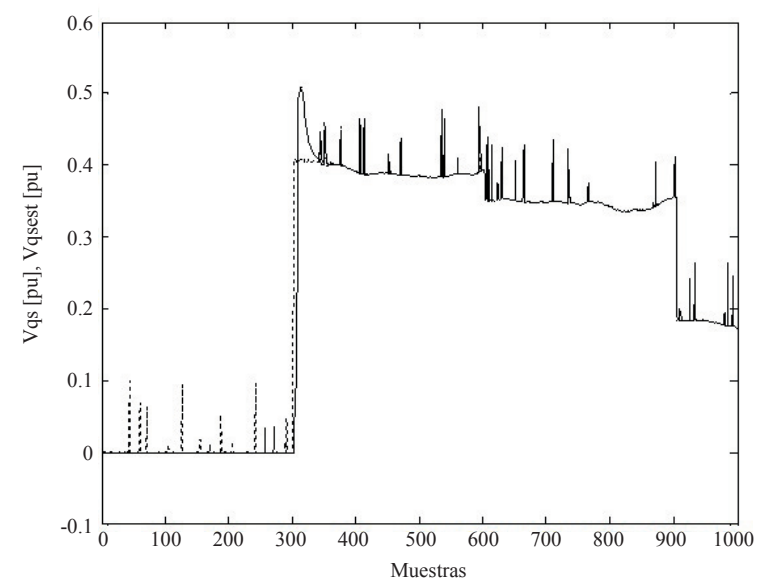

Figura 6. Comportamiento del voltaje del estator en eje en cuadratura en presencia de datos espurios empleando el $D K F$. (---) corresponde al voltaje de la máquina (Vqs) y (—) es el voltaje estimado (Vqsest) por el DKF.

También de las Figuras 7 y 8 , se puede notar que los datos espurios toman valores de 0.2 pu por encima del valor medido, esto equivale a una cantidad considerable, sin embargo, la metodología responde adecuadamente ante el problema de estimación de parámetros.

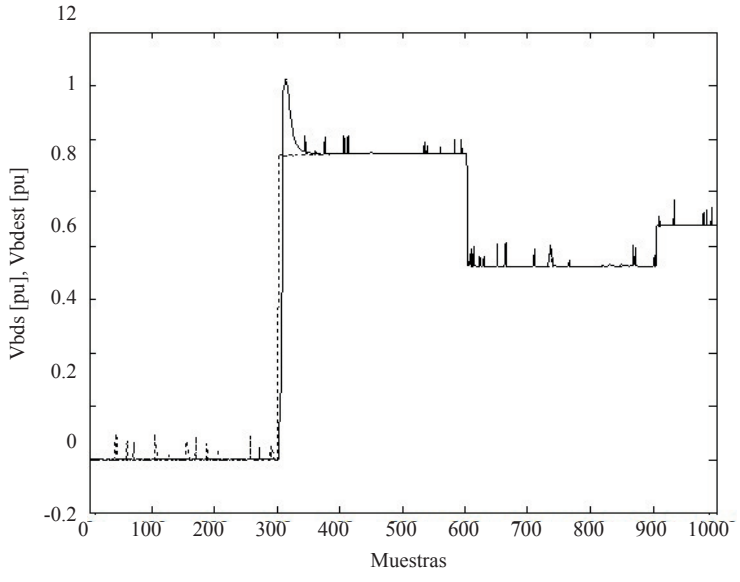

Figura 5. Comportamiento del voltaje del estator en eje directo en presencia de datos espurios empleando el DKF. (----) corresponde al voltaje de la máquina (Vds) y (-) es el voltaje estimado (Vdsest) por el DKF.

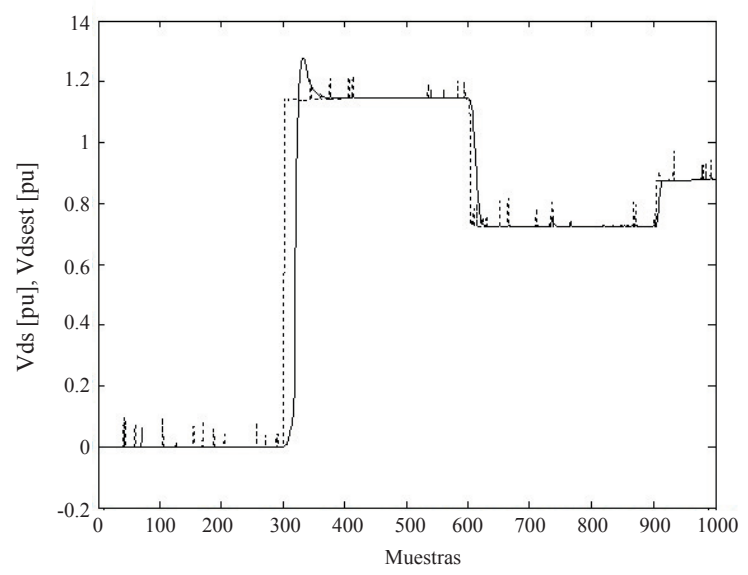

Figura 7. Comportamiento del voltaje en el estator en eje en cuadratura con datos espurios agregados. (---) corresponde al voltaje de la máquina (Vds) y (—) pertenece al voltaje estimado (Vdsest) por el DKF-rsKF.

En la Tabla 1, se encuentran consignados los errores residuales, usando , para el caso de la identificación del DFIG con el DKF y el DKF-rsKF. De esta tabla se muestra que el algoritmo de identificación propuesto alcanza errores del $0.51 \%$ comparado con los alcanzados por la técnica estándar que obtiene 


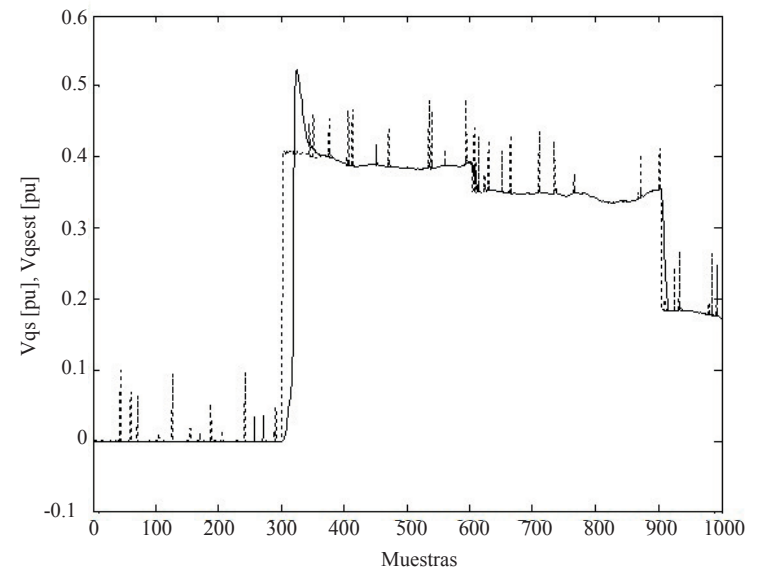

Figura 8. Comportamiento del voltaje en el estator en eje directo con datos espurios agregados. (---) corresponde al voltaje de la máquina (Vqs) y (_) pertenece al voltaje estimado (Vqsest) por el DKF-rsKF.

Tabla 1. Error residual para el filtro de Kalman dual estándar y la combinación del filtro de Kalman dual con el filtro de Kalman de estadística robusta; medida dada en escala absoluta.

\begin{tabular}{cc}
\hline & $\mathrm{E}_{\text {res }}$ \\
\hline DKF & $0.0848 \pm 0.0131$ \\
DKF-rsKF & $0.0051 \pm 0.0019$ \\
\hline
\end{tabular}

un error del $8.48 \%$, cuando se tiene el $5 \%$ de datos espurios en la señal.

Con el fin de analizar el desempeño del algoritmo propuesto, se realiza variaciones del nivel de ruido presente en las observaciones, donde las variaciones corresponden a relaciones señal a ruido de $5 \mathrm{~dB}, 10 \mathrm{~dB}, 15 \mathrm{~dB}, 20 \mathrm{~dB}, 25 \mathrm{~dB}$, y 30 $\mathrm{dB}$; se procedió a observar el efecto del ruido en la señal estimado por la técnica propuesta, para esto se calculó el error residual entre las observaciones y las predicciones respectivas.

Por lo tanto en la Figura 9, se puede notar que a medida que se decrementa el nivel de ruido también lo hace el error residual, se observa que para el caso más crítico se obtiene un error del $1.2 \%$ para un nivel de ruido del $30 \%(5 \mathrm{~dB})$

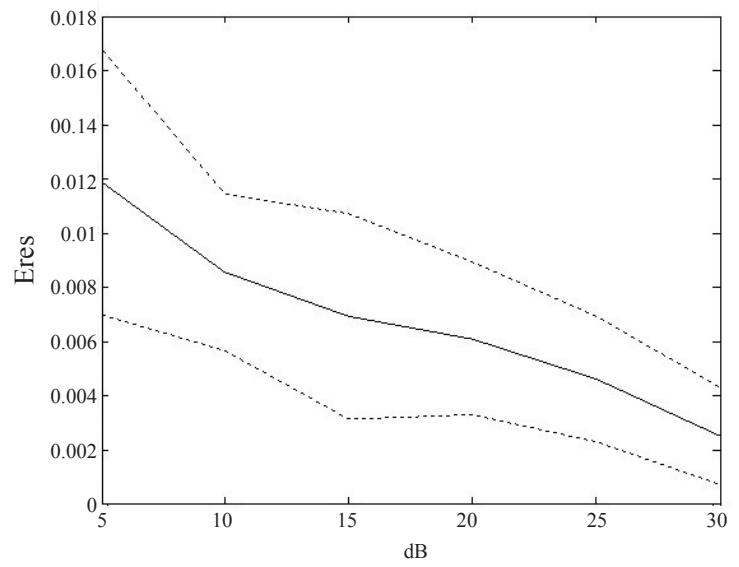

Figura 9. Análisis de robustez del DFIG empleando el método propuesto para diferentes relaciones señal a ruido dadas en decibel (dB); (-) corresponde a la media del error residual en escala absoluta; $y$ (----) corresponden a más o menos una desviación estándar alrededor de la media.

\section{Conclusiones}

En este estudio se planteó la combinación de dos técnicas para la identificación de una generador de inducción doblemente alimentado dando robustez a datos espurios, la primer técnica equivale a tener el filtro de Kalman dual y la otra equivale a una variación del filtro de Kalman estándar (rsKF); en donde el método propuesto mostro que no se vio afectado con la presencia de estos datos espurios obteniendo errores menores al 1.2\%; Para el caso de tener una aplicación de generación de energía la presencia de estos datos afectaría la confiabilidad del sistema de potencia a la cual esté conectado el DFIG.

Igualmente se pudo notar que la metodología se puede aplicar en estudios de estimación de parámetros en línea, que hace que estas técnicas de identificación sean de gran uso; también se debe destacar que la metodología es de fácil implementación en ambientes prácticos.

También es pertinente señalar que la etapa de estimación dual es importante para llevar a cabo la identificación del sistema y al mismo tiempo se debe mencionar que esta etapa no se ve perturbada 
por la presencia de estos datos que son un gran problema en aplicaciones de la ingeniería.

\section{Agradecimientos}

Este trabajo se desarrolló en el marco del proyecto de investigación: "Identificación de parámetros de un generador eólico doblemente alimentado basado en métodos recursivos bayesianos" en el programa de jóvenes investigadores "Virginia Gutiérrez de Pineda" y fue financiado por medio de Colciencias y la Universidad Tecnológica de Pereira.

\section{Referencias bibliográficas}

Anderson, B. \& Moore, J.B. (2005). Optimal Filtering (Dover Books on Engineering). Dover Publications.

Barrero, F., Perez, J., Millan, R., \& Franquelo, L.G. (1999). Self-commissioning for voltagereferenced voltage-fed vector controlled induction motor drives. In Industrial Electronics Society, 1999. IECON '99 Proceedings. The 25th Annual Conference of the IEEE 3(3), 1033-1038.

Belfedal, C., Gherbi, S., Sedraoui, M., Moreau, S., Champenois, G., Allaoui, T., \& Denaï, M. A. (2010). Robust control of doubly fed induction generator for stand-alone applications. Electric Power Systems Research, 80(2), 230 - 239.

Bertoluzzo, M., Buja, G.S., \& Menis, R. (1997). Inverter voltage drop-free recursive least-squares parameter identification of a pwm inverterfed induction motor at standstill. In Industrial Electronics, 1997. ISIE'97., Proceedings of the IEEE International Symposium, 2, 649-654.

Bim, E. (2001). Fuzzy optimization for rotor constant identification of an indirect foc induction motor drive. Industrial Electronics, IEEE Transactions, 48(6), 1293 -1295.

Bishop C. (2006). Pattern recognition and machine learning (Information Science and Statistics). Springer-Verlag New York, Inc.
Borgard, D.E., Olsson, G., \& Lorenz, R.D. (1995). Accuracy issues for parameter estimation of field oriented induction machine drives. Industry Applications, IEEE Transactions, 31(4), 795-801.

Chai, H., \& Acarnley, P.P. (1992). Induction motor parameter estimation algorithm using spectral analysis. Electric Power Applications, IEE Proceedings $B, 139(3), 165-174$.

Cipra, T., \& Romera, R. (1997). Kalman filter with outliers and missing observations. TEST: An Official Journal of the Spanish Society of Statistics and Operations Research 6(2), 379-395.

Eleazer, W. (2011). Launch failures: the "oops!" factor. http://www.thespacereview.com/ article/1768/1

Endusa, B., Tomonobu, S., Naomitsu, U., Atsushi, Y., Hiroshi, K., \& Toshihisa, F. (2007). Gain scheduling control of variable speed wtg under widely varying turbulence loading. Renewable Energy ,32(14), 2407 - 2423.

Finch, J.W., Atkinson, D.J., \& Acarnley, P.P. (1998). Full-order estimator for induction motor states and parameters. Electric Power Applications, IEE Proceedings 145(3), 169-179.

Goodwin, G.C., \& Sin, K.S. (2009). Adaptive Filtering Prediction and Control. Dover Publications, Inc., New York, NY, USA.

Griva, G., Ficarra, M.C., \& Profumo, F. (1997). Design of a speed regulator for induction motor drives based on model reference robust control. In Industrial Electronics, 1997. ISIE '97., Proceedings of the IEEE International Symposium, 2, $485-488$.

Han, J., \& Kamber, M. (2000). Data Mining: Concepts and Techniques. Morgan Kaufmann, 1st edition.

Haykin S. (2001). Kalman Filtering and Neural Networks. Wiley-Interscience. 
Hofmann, W., \& Liang, Q. (1995). Parameter adaption for field-oriented ac-drives using neural network. In Neural Networks, 1995. Proceedings., IEEE International Conference, 5, 2332 -2337.

Huber, P.J. (1964). Robust estimation of a location parameter. Annals of Mathematical Statistics 35(1), 73-101.

Huber, P.J. (2009). Robust statistic. $2^{\text {nd }}$ Edition, Wiley Series in Probability and Statistic. New York.

Ljung, L. (1999). System Identification: Theory for the User. Prentice Hall, 2 editions.

Loron, L., \& Laliberte, G. (1993). Application of the extended kalman filter to parameters estimation of induction motors. In Power Electronics and Applications, 1993, Fifth European Conference, $5,85-90$.

Masreliez, C. (1975). Approximate non-Gaussian filtering with linear state and observation relations. Automatic Control, IEEE Transactions, 20(1), $107-110$.

Masreliez, C., \& Martin, R. (1977). Robust Bayesian estimation for the linear model and robustifying the kalman filter. Automatic Control, IEEE Transactions, 22(3), 361-371.

Matsuo, T., \& Lipo, T.A. (1985). A rotor parameter identification scheme for vector-controlled induction motor drives. Industry Applications, IEEE Transactions, 21(3), 624-632.

Meinhold, R.J., \& Singpurwalla, N. D. (1989). Robustification of kalman filter models. Journal of the American Statistical Association, 84(406), $479-486$.

Moore, M., \& Mccabe, G. (1989). Introduction to the Practice of Statistics. Freeman, New York.

Ohnishi, K., Ueda, Y., \& Miyachi, K. (1986). Model reference adaptive system against rotor resistance variation in induction motor drive. Industrial Electronics, IEEE Transactions, 33(3), $217-223$.
Ruff, M., \& Grotstollen, H. (1993). Identification of the saturated mutual inductance of an asynchronous motor at standstill by recursive least squares algorithm. In Power Electronics and Applications, 1993., Fifth European Conference, $5,103-108$.

Ruff, M., Bunte, A., \& Grotstollen, H. (1994). A new self-commissioning scheme for an asynchronous motor drive system. In Industry Applications Society Annual Meeting, 1994., Conference Record of the 1994 IEEE 1, 616-623.

Sánchez, S., \& Giraldo, E. (2009). Sistemas de control en modelos de máquinas eléctricas. U.T.P. and ITM.

Schick, I. C., \& Mitter, S. K. (1994). Robust recursive estimation in the presence of heavytailed observation noise. The Annals of Statistics 22(2), 1045-1080.

Schierling, H. (1988). Self-commissioning-a novel feature of modern inverter-fed induction motor drives. In Power Electronics and VariableSpeed Drives, Third International Conference on $287-290$.

Smith, A.F., \& West, M. (1983). Monitoring renal transplants: an application of the multiprocess Kalman filter. Biometrics 39(4), 867-878.

Sorenson, H.W., \& Alspach, D.L. (1971). Recursive bayesian estimation using gaussian sums. Automatica 7(4), $465-479$.

Sørensen, P., Hansen, A.D., \& Carvalho, P.A. (2002). Wind models for simulation of power fluctuations from wind farms. Journal of Wind Engineering and Industrial Aerodynamics, 90(1215), $1381-1402$.

Sumner, M., \& Asher, G.M. (1993). Autocommissioning for voltage-referenced voltage-fed vector-controlled induction motor drives. Electric Power Applications, IEE Proceedings B 140(3), 187 -200. 
Ting, J.A., Theodorou, E., \& Schaal S. (2007a). A kalman filter for robust outlier detection. In Intelligent Robots and Systems, 2007. IROS 2007. IEEE/RSJ International Conference on 1514-1519.

Ting, J.-A., D’Souza, A., \& Schaal, S. (2007b). Automatic outlier detection: A bayesian approach. In Robotics and Automation, 2007 IEEE International Conference on 2489-2494.

Ting, J.-A. (2009). Bayesian methods for autonomous learning systems. Doctoral thesis, Los Angeles, CA, USA.

Toliyat, H.A., \& Hosseiny, A.A.Gh. (1993). Parameter estimation algorithm using spectral analysis for vector controlled induction motor drives. In Industrial Electronics, 1993. Conference Proceedings, ISIE'93 Budapest., IEEE International Symposium, 90 -95.

Tukey, J.W. (1960). A survey of sampling from contaminated distributions. Contributions to Probability and Statistics Essays in Honor of Harold Hotelling 448-485.

West, M. (1981). Robust sequential approximate bayesian estimation. Journal of the Royal Statistical Society. Series B (Methodological) 43(2), 157-166.
Zai, L.-C., DeMarco, C.L., \& Lipo, T.A. (1992). An extended kalman filter approach to rotor time constant measurement in pwm induction motor drives. Industry Applications, IEEE Transaction, 28(1), $96-104$.

ApéndiceA. Datos del generador de inducción doblemente alimentado

La Tabla 2 presenta el conjunto de parámetros de un generador de inducción doblemente alimentado de $9 \mathrm{MW}, 25 \mathrm{kV}$ y $60 \mathrm{~Hz}$.

Tabla 2. Conjunto de parámetros de un generador de inducción doblemente alimentado de $9 \mathrm{MW}, 25 \mathrm{kV}$ y $60 \mathrm{~Hz}$

\begin{tabular}{lc}
\hline$R_{s}$ & $0.00706(\mathrm{pu})$ \\
$R_{r}$ & $0.005(\mathrm{pu})$ \\
$L_{m}$ & $2.9(\mathrm{pu})$ \\
$L_{s}$ & $0.171[\mathrm{pu}]$ \\
$L_{r}$ & $0.156[\mathrm{pu}]$ \\
$H$ & $5.04[\mathrm{~s}]$ \\
$p$ & 3 \\
$\omega_{s}$ & $1[\mathrm{pu}]$ \\
\hline
\end{tabular}

